\title{
Warm inflation as a way out of the swampland
}

\author{
Meysam Motaharfar,, , f Vahid Kamali, ${ }^{2}$, 闹 and Rudnei O. Ramos ${ }^{3}$, 用 \\ ${ }^{1}$ Department of Physics, Shahid Beheshti University, G. C., Evin,Tehran 19839, Iran \\ ${ }^{2}$ Department of Physics, Bu-Ali Sina University, Hamedan 65178, 016016, Iran \\ ${ }^{3}$ Departamento de Fisica Teorica, Universidade do Estado do Rio de Janeiro, 20550-013 Rio de Janeiro, RJ, Brazil
}

\begin{abstract}
We discuss how dissipative effects and the presence of a thermal radiation bath, which are inherent characteristics of the warm inflation dynamics, can evade the recently proposed Swampland conjectures. Different forms of dissipation terms, motivated by both microphysical quantum field theory and phenomenological models, are discussed and their viability to overcome the assumed Swampland constraints is analyzed.
\end{abstract}

\section{INTRODUCTION}

The cosmological observational data unanimously confirm that the Universe is expanding, spatially flat, homogeneous, and isotropic on large scales and the Large Scale Structure (LSS) originated from the inhomogeneities of primordial origin, which are adiabatic and Gaussian and with a quasi-invariant scale power spectrum 11. The current paradigm for the evolution of the early Universe, cosmological inflation [2], consisting of a short period of quasi-de Sitter accelerated expansion phase, is able to successfully explain several puzzles of the standard big bang cosmology. Inflation solves the horizon and flatness problems and at the same time accounts for the origin of the inhomogeneities in the Universe able to lead to the LSS formation, based on causal physics. Although inflation generally occurs when the inflaton field is superPlanckian, especially when considering monomial chaotic potentials, when the currently observable scale exits the Hubble horizon, the energy scale is much smaller than the Planck scale. Therefore, it is assumed that inflation can be well described by some low-energy effective theory (EFT). However, this does not mean that any inflationary model, in the language of the effective theory, can be ultraviolet complete. In fact, consistently embedding the EFT of gravity into a quantum theory, in particular in the context of string theory, requires distinguishing consistent low-energy EFT coupled to gravity from inconsistent counterparts. The latter are deemed to inhabit the so-called Swampland 3, 4. Hence, having correct criteria to identify the boundary between the landscape and the Swampland has resulted in a series of conjectures known as weak gravity [5] and Swampland conjectures [6], motivated by black hole physics 7 and string compactification [8]. Recently, it was proposed that an effective field theory to be consistently embedded in quantum gravity must satisfy these conjectures. These conjectures then turn out to lead to constraints in the allowed range of field excursion and the potential field gradient. In turn,

\footnotetext{
*Electronic address: mmotaharfar2000@gmail.com

${ }^{\dagger}$ Electronic address: vkamali@basu.ac.ir

${ }^{\ddagger}$ Electronic address: rudnei@uerj.br
}

these conjectures have lead to severe constraints on the possible inflation models able to be described in terms of effective models of inflation [9. The two Swampland conjectures are as follows.

- The Swampland distance conjecture $(S D C)$.- The scalar field excursions in field space are bounded from above [4:

$$
\frac{|\Delta \phi|}{M_{\mathrm{Pl}}}<\Delta \sim \mathcal{O}(1),
$$

where $\Delta$ is some constant of the order of unity, that depends on the details of the string model compactification. $M_{\mathrm{Pl}} \equiv(8 \pi G)^{-1 / 2} \simeq 2.4 \times 10^{18} \mathrm{GeV}$ is the reduced Planck mass.

- The Swampland de Sitter conjecture (SdSC).- The field gradient of the potential $V$ (with $V>0$ ) in any consistent theory of gravity should satisfy the lower bound [6]:

$$
M_{\mathrm{Pl}}\left(\frac{\left|\nabla_{\phi} V\right|}{V}\right)>c \sim \mathcal{O}(1),
$$

with $c$ being another constant also of the order of unity.

It should be noticed that Eq. (1.2) not only requires steep potentials but also forbids models including extrema or plateaus, for which $\nabla_{\phi} V / V \rightarrow 0$ in one or more points in field space. Although the authors in Ref. 10] have shown that there is still controversy on the specific value for $c$, which may make the discussion irrelevant, we will assume Eq. (1.2) for the sake of argument.

Hence, by taking at face value and by assuming the validity of the above two conjectures, they may have serious cosmological implications as discussed by many authors recently 9, 11, in particular, for inflationary physics [12, 13. In fact, possible inflation theories, especially slow-roll single field inflation, are tightly constrained. Thus, more complicated inflation models, or nonstandard physics of the early Universe, such as chromonatural inflation [14, multifield inflation [15], or curvaton scenarios [16] seem to be preferred by these two conjectures. One should notice that the validity of the above Swampland conjectures has also been questioned recently [17, 18. Independent of the real applicability or validity of these conjectures, we believe it is still worth seeking which inflationary model realizations or pictures 
might overcome them. This, in particular, might teach us something about the possible realization of these models in the context of effective field theories and a possible connection of them with more fundamental theories, like string theory. In view of these motivations, in this work, by assuming the validity of the above two Swampland conjectures, we would like to discuss them in the context of the warm inflation picture [19] as a possible way of realizing in a satisfactory way the conditions given by Eqs. (1.1) and (1.2). As we are going to show, by accounting for the nonequilibrium dissipative processes emerging from the microscopic dynamics of the inflaton field during warm inflation, which can produce a quasiequilibrium thermal radiation bath during inflation (for reviews on warm inflation, see, e.g., Refs. [20, 21]), the dynamics of inflation can change considerably at both the background and perturbation levels compared with the case where these effects are neglected. Consequently, warm inflation can lead naturally to different consistency relations compared to the case when these dissipative effects are negligible, like in the usual cold inflation scenario. Thus, warm inflation can possibly lead to conditions able, in principle, to satisfy Eqs. (1.1) and (1.2). This ability for warm inflation possibly evading the Swampland conditions was first noticed in Ref. [22]. In this work we would like to establish whether warm inflation is indeed able to satisfy Eqs. (1.1) and (1.2) and under which conditions and for which possible models this can indeed happen.

This paper is organized as follows. In Sec. II] we briefly review the warm inflation dynamics and its relevant equations, which we will be using in this work. Next, in Sec. III] we will then study different dissipation mechanisms that can lead to the warm inflation dynamics, either directly motivated from particle physics realizations or phenomenological motivated ones. We will then discuss these models in the context of the Swampland conjectures. Finally, in Sec. [V] we give our conclusions and final comments.

\section{WARM INFLATION DYNAMICS}

The dynamics of inflation can go through two different pictures: cold inflation (CI) and warm inflation (WI), depending on whether nonequilibrium dissipative processes due to the couplings of the inflaton field with other field degrees of freedom are negligible or not during inflation. In fact, dissipation processes determine how ultimately the vacuum energy density, stored in the inflaton field, ends up converting into radiation, thus allowing the Universe to make a transition from the accelerating phase to the radiation-dominated epoch. The first and more conventional picture for inflation is the isentropic CI, where the inflaton field can be treated as essentially isolated from interacting with other subdominant quantum fields, whereby any previous preinflationary radiation energy density drastically decreases. Later, at the end of inflation, the inflaton starts oscillating around the mini- mum of its potential and progressively dissipates its energy into other relativistic light degrees of freedom that thermalizes and provides the radiation bath required by the standard big bang cosmology. Thus, CI necessarily requires a phase of (p)reheating (for some recent reviews on the (p)reheating theory, see, e.g., Refs. 223, 24, and references therein). In contrast to this picture, in WI, the inflaton interactions with other subleading quantum fields can be strong enough in such a way that their effects may not become negligible. Therefore, the state of the inflationary universe can become one not that of a quasiperfect vacuum state like in CI, but rather an excited statistical state, with a thermal state being the most examined [20, 21]. Even so, in WI the vacuum energy is still the dominant component for the accelerated expansion to take place. Consequently, non-negligible dissipative processes can take place not only after but also during the slow-roll phase of inflation, whereby a quasiequilibrium thermal radiation bath is concurrently generated throughout inflation. This state can be such to compensate the supercooling phase observed in CI and radiation can smoothly be produced and it becomes the dominant energy component of the Universe at the end of the inflationary expansion.

The total energy density in WI is then given by

$$
\rho=\frac{\dot{\phi}^{2}}{2}+V(\phi)+\rho_{R},
$$

which accounts for the presence of the radiation fluid, with energy density $\rho_{R}$, and the scalar field (the inflaton) $\phi$, with some potential $V(\phi)$. The inflaton field $\phi$ and the radiation energy density $\rho_{R}$ form a coupled system in WI dynamics, with background evolution equations given, respectively, by

$$
\begin{aligned}
& \ddot{\phi}+3 H \dot{\phi}+\Upsilon(\phi, T) \dot{\phi}+V_{, \phi}=0, \\
& \dot{\rho}_{R}+4 H \rho_{R}=\Upsilon(\phi, T) \dot{\phi}^{2},
\end{aligned}
$$

where $\Upsilon(\phi, T)$ is the dissipation coefficient in WI, which can be a function of the temperature and/or the background inflaton field. We will define below explicitly the dissipation coefficient forms we will be considering in this work. For a radiation bath of relativistic particles, the radiation energy density is given by $\rho_{R}=\pi^{2} g_{*} T^{4} / 30$, where $g_{*}$ is the effective number of light degrees of freedom $\left(g_{*}\right.$ is fixed according to the dissipation regime and interactions form used in WI). In WI, it is usual to define the ratio of the dissipation coefficient with the Hubble rate $H$ as

$$
Q=\frac{\Upsilon(T, \phi)}{3 H},
$$

which in some sense parametrizes the strength of the WI dissipative processes. The dissipation coefficient $\Upsilon$ in Eqs. 2.2 and (2.3) embodies the microscopic physics resulting from the interactions between the inflaton and the other fields that can be present and accounts for the nonequilibrium dissipative processes arising from these 
interactions. The dissipation coefficient $\Upsilon$ is, in general, well defined in the context of nonequilibrium quantum field theory methods [20, 25, 26]. The effect of the dissipation term in the above equations can be such as to be able to sustain a temperature $T \geq H$ throughout inflation for $\dot{\phi} \gg H^{2}$, even for $\Upsilon \ll H$ (i.e., $Q \ll 1$ ), without violating the slow-roll conditions. In particular, the additional friction term in Eqs. 2.2 and (2.3) modifies the usual slow-roll conditions to $\left\{\epsilon_{V}, \eta_{V}\right\} \ll 1+Q$, where $\epsilon_{V}=M_{\mathrm{Pl}}^{2}\left(V_{, \phi} / V\right)^{2} / 2$ and $\eta_{V}=M_{\mathrm{Pl}}^{2} V_{, \phi \phi} / V$ are the usual (inflaton potential) slow-roll parameters. Therefore, the conventional Hubble slow-roll parameter $\epsilon_{H}$ changes to

$$
\epsilon_{H}=-\frac{\dot{H}}{H^{2}} \simeq \frac{\epsilon_{V}}{1+Q} .
$$

Thus, the accelerated inflationary dynamics terminates when $\epsilon_{H}=1$ or, equivalently, when $\epsilon_{V}=1+Q$. Moreover, the ratio of radiation to inflaton energy density in the slow-roll regime is roughly given by

$$
\frac{\rho_{R}}{\rho_{\phi}} \simeq \frac{1}{2} \frac{\epsilon_{V}}{1+Q} \frac{Q}{1+Q} .
$$

Therefore, during inflation, the energy density associated with the inflaton field still dominates over the one from the radiation, $\rho_{\phi} \gg \rho_{R}$. Hence, radiation is somewhat still suppressed, as we should expect. Even though this condition might be satisfied for weak dissipation $(Q \ll 1)$, at the end of inflation for $\epsilon_{V} \sim 1+Q$ and $\rho_{R} / \rho_{\phi} \simeq(1+Q) Q / 2$, radiation can become the dominant component. Consequently, the Universe can smoothly enter into a radiation-dominated era without the need of a reheating phase a priori. In fact, steep potentials without extrema and plateau can also be embedded in WI, as the Swampland criteria typically prefer.

\section{A. The Swampland and a lower bound on the value of the dissipation}

Slow-roll CI models are in tension with the SdSC, since in such models $\epsilon_{H} \simeq \epsilon_{V}$ and Eq. 1.2 would imply that $\epsilon_{V}>c^{2} / 2$, thus preventing an accelerating expansion. Even though the condition $c>1$ can, in principle, be relaxed [16, one notices that WI can break the approximate relation between $\epsilon_{H}$ and $\epsilon_{V}$ as discussed above, from Eq. (2.5), allowing accelerated expansion even if $\epsilon_{V}>1$, provided that $Q>1$. One notes also the SdSC condition, Eq. $(1.2)$, targets the heart of CI models based on the requirement of the standard (p)reheating mechanism, which usually occurs around the minimum of the potential, $V_{, \phi}=0$. As already discussed above, the continuous radiation production in WI due to the dissipative effects can naturally replace the usual reheating mechanism by smoothly ending inflation already in a radiationdominate regime. Thus, WI does not require, in general, a potential with a minimum or that $V_{, \phi}=0$ as a condition for reheating. Furthermore, note that we can also express the SDC condition, Eq. 1.1, for the field excursion in terms of the slow-roll parameter $\epsilon_{V}$ and the number of $e$-folds $N$ as (using $d N=H d t$ )

$$
\frac{\Delta \phi}{M_{\mathrm{Pl}}}=\frac{\dot{\phi}}{H} N \simeq \frac{\sqrt{2 \epsilon_{V}}}{(1+Q)} N .
$$

Note that in CI $(Q \equiv 0)$ we can have plateau-type potentials for which $\epsilon_{V} \ll 1$ and $N \gg 60$, which is more than necessary for solving the usual big bang cosmological problems, leading to very small field excursions and thus satisfying the condition Eq. 1.1). However, plateau-type potentials are strongly disfavored by the second Swampland condition Eq. (1.2). Even so, we see that the change of the slow-roll parameters by the dissipation ratio $Q$ in Eq. (2.7) offers again a way out of the SdSC condition if $Q \gg 1$ and $N$ is still within the required number of $e$-folds, $N \sim 60$. Thus, putting these conditions together in the context of WI, such as to simultaneously satisfy both conjectures, we are lead to a lower bound on the dissipation ratio $Q$ as given by

$$
Q>\frac{c}{\Delta} N-1
$$

We can regard Eq. 2.8 as a general lower bound on $Q$ for WI models, regardless of the exact value of $c$ and $\Delta$, to be able to simultaneously satisfy both Swampland conjectures. Although there is still an uncertainty on the exact values for $c$ and $\Delta$, the above bound shows that $\mathrm{WI}$ is on the landscape, given an appropriate value for the dissipation ratio $Q$, and even for $c$ and $\Delta$ taken to be of the order of unity and with $N \sim 60$. By relaxing these parameters, we can also make it easier for warm inflation to satisfy the Swampland conjectures for smaller values of $Q$, as we will show in Sec. III below.

\section{B. The Swampland, WI, and the scalar and tensor perturbations}

In WI, not only the background dynamics gets modified by the dissipative effects, but also the perturbations get modified due to the presence of dissipation and the radiation bath. Especially, the primordial power spectrum can be strongly influenced by these effects (see, e.g., Refs. [27 32]). For instance, the primordial power spectrum for WI at horizon crossing can be expressed in the form (see, e.g., Ref. 33] and references therein)

$$
\Delta_{\mathcal{R}}\left(k / k_{*}\right)=P_{0}\left(k / k_{*}\right) \mathcal{F}\left(k / k_{*}\right),
$$

where we indicate with a subindex "*" those quantities evaluated at the horizon crossing. The contribution $P_{0}\left(k / k_{*}\right)$ in Eq. 2.9) is the standard CI result:

$$
P_{0}\left(k / k_{*}\right) \equiv\left(\frac{H_{*}^{2}}{2 \pi \dot{\phi}_{*}}\right)^{2},
$$


while $\mathcal{F}\left(k / k_{*}\right)$ in Eq. 2.9 is the WI modification to the primordial power spectrum:

$$
\mathcal{F}\left(k / k_{*}\right) \equiv\left(1+2 n_{*}+\frac{2 \sqrt{3} \pi Q_{*}}{\sqrt{3+4 \pi Q_{*}}} \frac{T_{*}}{H_{*}}\right) G\left(Q_{*}\right) .
$$

In the latter equation, $n_{*}$ denotes the inflaton statistical distribution due to the presence of the radiation bath. For a thermal equilibrium distribution, it assumes the Bose-Einstein distribution form, $n_{*}=1 /\left[\exp \left(H_{*} / T_{*}\right)-\right.$ 1]. The function $G\left(Q_{*}\right)$ in Eq. 2.11) accounts for the effect of the coupling of the inflaton fluctuations with radiation 28 30, which we will specify in more detail in the next section below. The scalar spectral amplitude value at the pivot scale $k_{*}$ is set by the cosmic microwave background $(\mathrm{CMB})$ radiation data as $\Delta_{\mathcal{R}}(k=$ $\left.k_{*}\right) \simeq 2.2 \times 10^{-9}$, with $k_{*}=0.05 \mathrm{Mpc}^{-1}$ as considered, e.g., by the Planck Collaboration [1].

While the primordial scalar curvature perturbation in WI gets modified according to Eq. 22.9p, the tensor perturbations spectrum is simply of the CI form, $\Delta_{T}=2 H_{*}^{2} /\left(\pi^{2} M_{p}^{2}\right)$. This is so because of the weakness of the gravitational interactions. Hence, the spectrum of primordial gravitational waves remains unaffected by the dissipative dynamics of WI 34] (see also Ref. 35] for a recent study on the possible changes of the tensor spectrum in WI). The tensor-to-scalar ratio $r$,

$$
r=\frac{\Delta_{T}}{\Delta_{\mathcal{R}}}
$$

leads now to a modified consistency relation in WI when compared to the CI result,

$$
r=\frac{16 \epsilon_{V}}{\left(1+Q_{\star}\right)^{2}} \mathcal{F}^{-1}\left(k / k_{*}\right) .
$$

Combining this result with Eq. 2.7) and the two Swampland conjectures Eqs. (1.1) and (1.2), we are lead to the following requirement:

$$
\frac{8 c^{2}}{(1+Q)^{2}} \frac{1}{\mathcal{F}}<r<\frac{8 \Delta^{2}}{N^{2}} \frac{1}{\mathcal{F}} .
$$

Note that for $Q=0$ (when $\mathcal{F} \rightarrow 1$ ), then Eq. 2.14 reduces to the relation obtained for CI when considering the Swampland conjectures [13. Although it is rather difficult for both constraints in Eq. 2.14 to be simultaneously satisfied in CI for $\{c, \Delta\} \sim \mathcal{O}(1)$, it can be possible to be arranged for both of them to be satisfied in WI. This can happen, for instance, if the dissipation ratio is larger than the $e$-folding number as we have already stated in Eq. (2.8). If we relax on the $c$ and $\Delta$ values, it can also be arranged for Eq. (2.14) to be satisfied even for smaller values of $Q$, as we will show in Sec. III. Besides, it is important to also check what the effects of such given values of $Q$, able to satisfy Eq. 2.14) as required by the Swampland conditions, might have on the observables and whether they would still be allowable when confronting some specific models with the observational data. This is what we now focus on below.

\section{SOME EXPLICIT MODEL REALIZATIONS AND OBSERVATIONAL CONSEQUENCES}

Dissipation processes during WI modify not only the homogeneous evolution of the inflaton field but also its inhomogeneous fluctuations. Therefore, the curvature power spectrum gets modified in different ways. First, it directly sources inflaton fluctuations, yielding a Langevin equation that generalizes Eq. (2.2) for an inhomogeneous field $27-32$. In fact, the source of primordial perturbations stems from thermal fluctuation in the radiation bath, which is then transferred to the inflaton field as adiabatic curvature perturbations. Hence, WI predicts that the origin of perturbations is thermal rather than quantum. Inflaton quanta may also be thermally excited during inflation and acquire a Bose-Einstein rather than a vacuum phase space distribution. Finally, inflaton and radiation fluctuations are, in general, coupled due to the possible explicit temperature dependence in the dissipative coefficient function $\Upsilon(\phi, T)$. This can be understood once the perturbations for WI are explicitly written down 28]. For example, if we parametrize the dissipation coefficient as

$$
\Upsilon(\phi, T)=C_{\phi} T^{s} / \phi^{s-1},
$$

where $s$ is some real number, the perturbed form for Eq. (3.1) becomes

$$
\delta \Upsilon=\left[\frac{s}{4} \frac{\delta \rho_{R}}{\rho_{R}}-(s-1) \frac{\delta \phi}{\phi}\right] \Upsilon,
$$

which explicitly makes the inflaton perturbations $\delta \phi$ to be coupled with those of the radiation bath $\delta \rho_{R}$. As shown in Ref. 28, this can generate in the curvature power spectrum a growing mode (if $s>0$ ) or a decreasing mode (if $s<0$ ), as the dissipation ratio $Q$ increases. For instance, the resulting dimensionless power spectrum in WI becomes Eq. (2.9), with $\mathcal{F}$ given by Eq. (2.11), and the function $G\left(Q_{*}\right)$ in that equation takes into account this effect of the coupling between inflaton and radiation perturbations. The strongest effect of this term on the observables manifests through the spectral tilt $n_{s}$ :

$$
n_{s}-1=\lim _{k \rightarrow k_{*}} \frac{d \ln \Delta_{\mathcal{R}}\left(k / k_{*}\right)}{d \ln \left(k / k_{*}\right)},
$$

which can be driven quickly to bluer values $\left(n_{s}>1\right)$ at larger values of $Q$ when $s>1$ or lead to a redder spectrum when $s<0$.

In our analysis below, we will consider five forms for the dissipation coefficient $\Upsilon$ that have been considered with some frequency before in the literature and which are motivated by the parameterization of the form of Eq. (3.1), namely

,(a) a dissipation coefficient with a cubic dependence in the temperature,

$$
\Upsilon_{\text {cubic }}=C_{\text {cubic }} T^{3} / \phi^{2} ;
$$


(b) a dissipation coefficient with a linear dependence in the temperature,

$$
\Upsilon_{\text {linear }}=C_{\text {linear }} T
$$

(c) a dissipation coefficient inversely proportional to the temperature,

$$
\Upsilon_{\text {inverse }}=C_{\text {inverse }} \phi^{2} / T
$$

(d) a dissipation coefficient that is constant,

$$
\Upsilon_{\text {constant }}=C_{\text {constant }} M_{\mathrm{Pl}} \text {; }
$$

and (e) a dissipation coefficient proportional to the Hubble parameter,

$$
\Upsilon_{\mathrm{H}}=C_{\mathrm{H}} H
$$

A dissipation coefficient of the form of $\Upsilon_{\text {cubic }}$, Eq. (3.4), emerges naturally for models of WI when the inflaton is coupled to heavy intermediate fields, that are in turn coupled to light radiation fields $20,25,26$. This is obtained in the so-called low-temperature regime for WI, in which the inflaton couples only to the heavy intermediate fields, whose masses are larger than the radiation temperature, $M \gg T$, and, thus, the inflaton gets decoupled from the radiation fields. The dissipation coefficient of the form of $\Upsilon_{\text {linear }}$, Eq. (3.5), has been explicitly constructed recently [36], and it is based on a construction used in Higgs phenomenology beyond the standard model, which uses a collective symmetry where the inflaton is a pseudoGoldstone boson. In this case, the inflaton is directly coupled to the radiation fields and gets protection from large thermal corrections due to the symmetries obeyed by the model. In these models, the dissipative coefficient is derived in the high-temperature regime, where the fields coupled to the inflaton are light with respect to the ambient temperature, $M<T$. The form of the dissipation coefficient as given by Eq. (3.6) originated in the first studies of warm inflation based on nonequilibrium quantum field theory methods [37, 38, and where it was also derived in a high-temperature regime, $M<T$. Though this type of model is more difficult to explicitly construct as compared to the two previous cases, we include it here as an example of a dissipation regime that can lead to a suppression of the power spectrum at larger $Q$ values and, thus, to a redder spectrum as compared to the cubic and linear in $T$ cases, which both tend to lead to a bluer spectrum at large $Q$. This is particularly important when setting limits on the dissipation ratio based on the spectral tilt $n_{s}$. As in WI we typically have that the temperature decreases as the end of inflation approaches, we will always have that eventually the high-temperature regime used to derive the dissipation coefficient like of the form of Eq. (3.6), will eventually transit to the lowtemperature form of Eq. (3.4) (see, e.g., Ref. [26]). This motivates us to study also a constant dissipation coefficient like in Eq. (3.7), which might describe this intermediate regime between the high- and low-temperature ones and if this regime lasts long enough. Finally, we also include a dissipation coefficient of the form of Eq. (3.8), since it leads strictly to a constant dissipation ratio $Q$. Such a form leading to a constant dissipation ratio is particularly useful for deriving analytical results in WI and has been employed by many authors before exactly because of that (see also Ref. [39] for other forms of dissipation coefficients). It can be seen as a sort of phenomenological dissipation form. In fact, many recent works dealing with dissipation effects in the recent Universe, like describing possible energy exchanges between the dark sectors, have been using this type of dependence (see, e.g., Ref. [40] and references therein), and it is also typically used to model dissipation effects in the form of effective viscosities as well [41.

Assuming for demonstrative purposes an inflaton potential given by a chaotic quartic potential ${ }^{1}, V(\phi)=$ $\lambda \phi^{4} / 4$, with coupling constant $\lambda$ fixed by the primordial scalar spectrum CMB amplitude according to the dissipation model used and spectrum given by Eq. (2.9). For the temperature-dependent dissipations, the function $G\left(Q_{*}\right)$ appearing in Eq. 2.11) can then be found to be [33]

$$
G_{\text {cubic }}\left(Q_{*}\right) \simeq 1+4.981 Q_{*}^{1.946}+0.127 Q_{*}^{4.330},
$$

for the cubic dissipation coefficient $\Upsilon_{\text {cubic }}$ and

$$
G_{\text {linear }}\left(Q_{*}\right) \simeq 1+0.335 Q_{*}^{1.364}+0.0185 Q_{*}^{2.315},
$$

for the linear dissipation coefficient $\Upsilon_{\text {linear }}$, while for the inverse in the temperature dissipation coefficient, $\Upsilon_{\text {inverse }}$, a similar numerical fitting as used to derive the previous two cases [29] leads to the result

$$
G_{\text {inverse }}\left(Q_{*}\right) \simeq \frac{1+0.4 Q_{*}^{0.77}}{\left(1+0.15 Q_{*}^{1.09}\right)^{2}} .
$$

For the dissipation terms independent of temperature, Eqs. (3.7) and (3.8), we can set this function $G$ equal to one. ${ }^{2}$

In Fig. 1, we show the results obtained for both $r$ and $n_{s}$ in WI for the five forms of dissipative coefficients given by Eqs. 3.4 - 3.8. For reference, for

\footnotetext{
1 Although strictly the form for this potential would not conform to the Swampland criterion SdSC, here we are interested only in the inflationary patch of the potential in the WI scenario. As also already explained before, we are not interested in describing (p)reheating here around the minimum of the potential, since in WI dynamics this is not required a priori. Thus, the dynamics around the minimum of the potential is not a concern to us here.

2 There is a weak temperature dependence in Eq. 3.8 through the radiation energy density contribution to the Hubble parameter, but during inflation, as already discussed, the dominant energy density component is that of the inflaton, so $H \propto \sqrt{V(\phi)}$ and we can still set $G_{\mathrm{H}} \sim 1$ with good accuracy.
} 


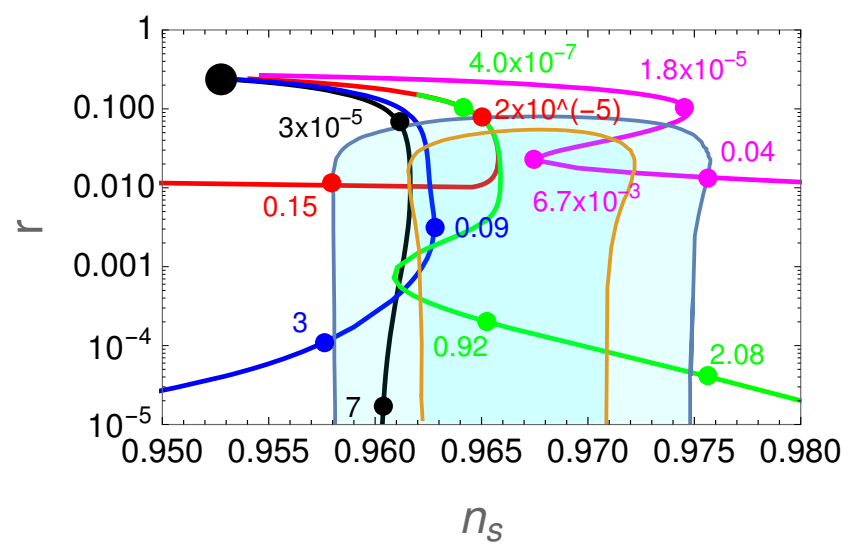

FIG. 1: The spectral index $n_{s}$ and the tensor-to-scalar ratio $r$ in the plane $\left(n_{s}, r\right)$ for different values of the dissipation ratio $Q_{*}$ (indicated by the numbers next to the curves) and for the different dissipation forms considered in the text: the dissipation coefficient proportional to $H$ (black line), the constant dissipation coefficient (red line), the inverse in $T$ dissipation coefficient (blue line), the linear in $T$ dissipation coefficient (green line), and the cubic in $T$ dissipation coefficient (magenta line). The large dot indicates the CI result $(Q=0)$. The contours are for the $68 \%$ and $95 \%$ C.L. results from Planck 2018 ( TT+TE+EE+lowE+lensing +BK14+BAO data).

each dissipation case, we have indicated some representative values of the dissipation ratio $Q_{*}$. The datasets used for the contours are for the $68 \%$ and $95 \%$ C.L. results from the recent Planck 2018 [42, and we have chosen the $\mathrm{TT}+\mathrm{TE}+\mathrm{EE}+$ lowE+lensing + BK14+BAO data, which is the most restrictive dataset. For definiteness, we have also considered $N_{*}=60$ to obtain the results shown in Fig. 1 .

Among the various dissipation cases studied, we see from Fig. 1 that the tensor-to-scalar ratio $r$ gets naturally suppressed quickly as $Q$ increases for all forms of dissipation terms considered, which is a natural feature of WI. However, the main limiting effect for going to larger values of $Q$ is on the spectral tilt. Figure 1 shows that most of the models typically do not allow for large values of $Q, Q>1$, with the maximum value reached for $Q_{*} \sim 2-3$, e.g., as in the cases of the linear and inverse in $T$ dissipation coefficients, beyond which compatibility with the Planck results for $n_{s}$ is jeopardized. These results are also compatible with earlier ones, obtained, for instance, in Ref. 33, when other forms of an inflaton potential were considered. An exception is for the constant dissipation ratio case, shown by the black curve in Fig. 1 . It does allow for fairly large values of $Q$. For instance, for $Q=100$, we have $n_{s} \simeq 0.96021$ and a fairly very small $r$ value, $r \simeq 1.1473 \times 10^{-7}$ and it is still inside the $95 \%$ C.L. contour for the Planck 2018 results.

As pointed out previously, there are models in WI 43 .

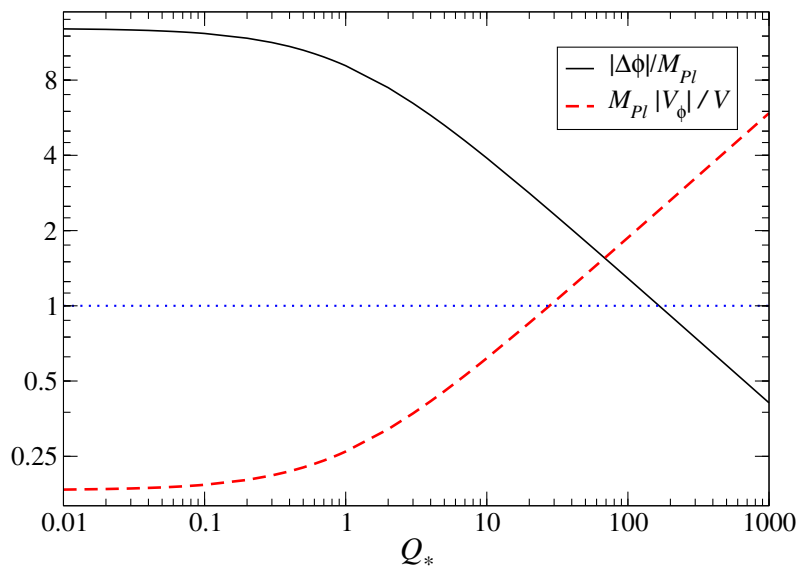

FIG. 2: The two Swampland conditions Eqs. 1.1 and 1.2 as a function of the dissipation ratio $Q_{*}$.

where we can find dissipation coefficients in a hightemperature regime (with respect to some mass scale, with $T \gg M$ ) which as the system evolves goes to a low-temperature $(T<M)$ regime. In these models, it becomes possible to arrange for a dissipation coefficient that can initially tend to lead to a very red spectrum for large $Q$, like the inverse in the temperature dissipation coefficient of Eq. (3.6), but that it ends up transiting at some point to a dissipation coefficient that tends to lead to a blue spectrum at large $Q$ values, like the cubic in the temperature form Eq. (3.4). In these cases it is possible that $Q$ can be bigger enough to comply with the bounds set by the Swampland conjectures. Even for the dissipation models studied here, if the Swampland-required values for $\Delta$ and $c$ in Eqs. (1.1) and 1.2 are slightly relaxed, this can make both conditions be satisfied already for values of $Q_{*}$ that are still within the Planck bounds shown in Fig. 1. In fact, there is quite some controversy on the precise values for $\Delta$ and $c$, which can also depend on the specific string model being considered [17]. As an example, in Fig. 2, we show the results for the two Swampland conditions as a function of $Q_{*}$ when taking the dissipation coefficient case given by Eq. (3.8). The other four dissipation coefficients show a similar evolution for these quantities. We see that, by relaxing the $\Delta$ value by a factor of around 4 bigger and decreasing the value of $c$ by also a factor of 4 smaller (both with respect to the unit value), we can already find values of the dissipation ratio, either in the linear or inverse in $T$ dissipation examples, satisfying both conditions and still have consistency with the Planck data.

Apart from the above results, one important thing to remark is that WI breaks the conventional Lyth bound 44, even for a small dissipation factor due to the presence of $(1+Q)^{-2} \mathcal{F}^{-1}$ in Eq. 2.13, resulting in a smaller tensor-to-scalar ratio $r$. This happens due to the effects of dissipation and the consequent deviation from the standard Bunch-Davies vacuum state (since, in WI, fluctuations are in an excited state). In fact, a de- 
viation from the standard Bunch-Davies state has been discussed as a possible way to save single-field slow-roll inflation against the Swampland conjectures [45]. One may also realize other mechanisms, like the proposal in Ref. 46], in order to produce a larger $c$ and use them in the context of WI so as to be able to suppress the value of $r$, allowing results to be consistent with observation and still having small $Q$. For instance, in brane inflation models [47, we have $\epsilon_{H}=\epsilon_{V} \frac{4 \Lambda}{V}$ with $\Lambda$ here denoting the brane tension. Then, in the regime where $V \gg \Lambda$, it results in $r=24 \epsilon_{H}$. Although the tensor-toscalar ratio $r$ can become larger in comparison with the standard CI, reconstructing it in the WI scenario allows one to make it consistent with observation [48] (see also Ref. 49] for a recent discussion of brane inflation and the Swampland). Moreover, all the kinetically modified models of inflation, such as $k$ inflation, including tachyon and Dirack-Born-Infeld models, $G$ inflation, and nonminimal derivative inflation, generally investigated as generalized $G$ inflation [50], result in an extra viscous term allowing $c$ to be larger. Although for larger $c$ they are in general, in the CI picture, in tension with observation, they can become consistent when constructed in the context of the WI scenario [51]. Therefore, even if the Swampland conjectures are strictly constrained to be unity, such an inherent characteristic of WI, i.e., a smaller energy scale, makes it as a way out of the Swampland.

\section{CONCLUSIONS}

In this work, we have studied the possibility of WI to satisfy the two recently proposed so-called Swampland conjectures. Despite the fact that there have been many discussions in the literature about the validity of these conjectures and how well they can indeed put constraints on existing models, particularly those involving inflation and dark energy, here we have assumed these conjectures to be valid and discussed WI in that context.

We recall that WI naturally predicts a lower tensorto-scalar ratio $r$ in essentially all models of inflation. But this becomes particularly relevant when considering monomial field chaotic inflation models, which are assumed already to be ruled out in the CI scenario. In particular, in the monomial $\phi^{4}$ model, this feature of WI in reducing $r$ has already been noticed for almost two decades [52]. This is of relevance to the Swampland conjectures, because that implies a lower scale of inflation in WI, when compared to CI, leading to a natural way for obtaining sub-Planckian inflaton field values during inflation, even for monomial chaotic inflation models. That the energy scale for inflation in WI can be smaller than that of CI for the same type of potentials has already been pointed out in Ref. [38. Also, in the review paper in Ref. [21, results for the tensor-to-scalar ratio were explicitly analyzed for monomial models, where it was also explicitly stated that WI predicts it to be smaller than in CI. This feature of having a lower $r$ in WI was then ex- plicitly found also in the more recent works [36, 53] and also when confronting WI with the CMB Planck data through explicit statistical analysis [33, 54]. By realizing inflation at lower energy scales, then WI makes it possible to drive different potential models within the safe region based on the Swampland conjectures.

Note added.- After completing this paper, a revised version of these Swampland conjectures was proposed in Ref. [55]. Besides the distance conjecture Eq. 11.1, in the refined de Sitter conjecture (RdSC), it is required that any scalar field potential from string theory should obey either Eq. 1.2 or $-M_{\mathrm{Pl}}^{2}\left(\nabla_{\phi}^{2} V\right) / V>c^{\prime}$, with $c^{\prime}$ being another constant also of the order of unity. This second condition from the refined conjecture is related, of course, with the definition of the slow-roll coefficient $\eta_{V}$ in cold inflation. As we have already mentioned in Sec. III, in WI the slow-roll condition on $\eta_{V}$ gets modified by the dissipation ratio $Q$ as $\eta_{V}<1+Q$. Except by the negative sign in the refined conjecture, which restricts the inflaton potential to be of the hilltop, small field type, we see that WI for large $Q$ values can still satisfy the refined version. In fact, this is related to how WI can provide a solution for the so-called $\eta$ problem, as already noticed long ago in the very early papers on WI (see, e.g., Ref. [52]).

Also, soon after completing this paper, another analysis of WI in the context of the Swampland conjectures appeared in Ref. [56. The author in that reference has reached, however, a different conclusion from us, stating that WI was already able to satisfy the conjectures even for small values of the dissipation ratio $Q$. The source of the difference can be traced back as a consequence of mistakenly comparing the upper bound coming from the observation for the tensor-to-scale ratio $(r<0.064)$ with the lower bound obtained from the de Sitter Swampland in Ref. [56]. More specifically, our inequality Eq. (2.14) is analogous to what is obtained when combining Eqs. (19) and (23) of Ref. [56. The difference is that the author there takes the function that we have called $\mathcal{F}$ in Eq. $2.9 p$ as $\sim \frac{T}{H} \sqrt{1+Q}$, when using an approximated (old) expression for the power spectrum in WI. Note that when considering the correct expression that we have used here, given by Eq. 2.9], and for the case of temperature-independent dissipation, we can then set $G(Q)=1$ in Eq. (2.11). Thus, for the case of $Q \ll 1$, we have $\mathcal{F} \sim 2 T / H$ (for $T / H \gg 1$, when still in the WI regime), and then our Eq. 2.14) becomes $4 c^{2}(H / T)<$ $r<4 \Delta^{2}\left(1 / N^{2}\right)(H / T)$. It then becomes obvious that it is quite hard for the inequalities to be simultaneously satisfied unless we relax severely either on $c$, on $\Delta$, or on both. However, for $Q \gg 1$, then $\mathcal{F} \sim \sqrt{3 \pi Q} T / H$ and now Eq. 2.14 becomes $\left(8 c^{2} / \sqrt{3 \pi}\right)(H / T)(1 / Q)^{5 / 2}<$ $r<\left(8 \Delta^{2} / \sqrt{3 \pi Q)}\left(1 / N^{2}\right)(H / T)\right.$. We can now understand why for large $Q$ both inequalities can be satisfied. This happens provided that $c^{2} / Q^{2}<\Delta^{2} / N^{2}$, which is actually just another way of writing Eq. (2.8). Furthermore, the results present here in Fig. 2 2 illustrate quite well the same result, independent of the derivation leading to Eq. (2.8) or Eq. 2.14. Moreover, it is worth mentioning that a 
different analysis performed recently by the authors of Ref. 57] has also reached a similar conclusion to ours.

\section{Acknowledgments}

R.O.R. is partially supported by research grants from Conselho Nacional de Desenvolvimento Científico e Tecnológico (CNPq), Grant No. 302545/2017-4, and Fundação Carlos Chagas Filho de Amparo à Pesquisa do Estado do Rio de Janeiro (FAPERJ), Grant No. E26/202.892/2017.
[1] N. Aghanim et al. (Planck Collaboration), Planck 2018 results. VI. Cosmological parameters, arXiv:1807.06209.

[2] A. A. Starobinsky, A new type of isotropic cosmological models without singularity, Phys. Lett. 91B, 99 (1980); K. Sato, First order phase transition of a vacuum and expansion of the universe, Mon. Not. R. Astron. Soc. 195, 467 (1981); A. H. Guth, The inflationary Universe: A possible solution to the horizon and flatness problems, Phys. Rev. D 23, 347 (1981); A. Albrecht and P. J. Steinhardt, Cosmology for grand unified theories with radiatively induced symmetry breaking, Phys. Rev. Lett. 48, 1220 (1982); A. D. Linde, A new inflationary Universe scenario: A possible solution of the horizon, flatness, homogeneity, isotropy and primordial monopole problems, Phys. Lett. 108B, 389 (1982).

[3] C. Vafa, The string landscape and the Swampland, arXiv:hep-th/0509212.

[4] H. Ooguri and C. Vafa, On the geometry of the string landscape and the Swampland, Nucl. Phys. B766, 21 (2007), arXiv:hep-th/0605264

[5] E. Palti, The weak gravity conjecture and scalar fields, J. High Energy Phys. 08 (2017) 034, arXiv:1705.04328

[6] G. Obied, H. Ooguri, L. Spodyneiko and C. Vafa, De Sitter space and the Swampland, arXiv:1806.08362.

[7] N. Arkani-Hamed, L. Motl, A. Nicolis, and C. Vafa, The string landscape, black holes and gravity as the weakest force J. High Energy Phys. 06 (2007) 060, arXiv:hepth/0601001

[8] H. Ooguri and C. Vafa, Non-supersymmetric AdS and the Swampland, Adv. Theor. Math. Phys. 21, 1787 (2017), arXiv:1610.01533; B. Freivogel and M. Kleban, Vacua morghulis, arXiv:1610.04564, T. D. Brennan, F. Carta, and C. Vafa, The string landscape, the Swampland, and the missing corner, Proc. Sci. TASI2017 (2017) 015, arXiv:1711.00864.

[9] P. Agrawal, G. Obied, P. J. Steinhardt, and C. Vafa, "On the cosmological implications of the string Swampland," Phys. Lett. B 784, 271 (2018), arXiv:1806.09718

[10] G. Dvali and C. Gomez, On exclusion of positive cosmological constant, Fortschr. Phys. 67, 1800092 (2019), arXiv:1806.10877.

[11] D. Andriot, New constraints on classical de Sitter: Flirting with the Swampland, Fortschr. Phys. 67, 1800103 (2019), arXiv:1807.09698, C. Roupec and T. Wrase, de Sitter extrema and the Swampland, Fortschr. Phys. 67, 1800082 (2019), arXiv:1807.09538 D. Andriot, On the de Sitter Swampland criterion, Phys. Lett. B 785, 570 (2018), arXiv:1806.10999 E. O. Colgain, M. H. P. M. Van Putten, and H. Yavartanoo, $H_{0}$ tension and the de Sitter Swampland, arXiv:1807.07451 L. Heisenberg, M. Bartelmann, R. Brandenberger, and A. Refregier, Dark en- ergy in the Swampland, Phys.Rev. D 98, 123502 (2018), arXiv: 1808.02877

[12] S. K. Garg and C. Krishnan, Bounds on slow roll and the de Sitter Swampland, arXiv:1807.05193; J. Brown, W. Cottrell, G. Shiu, and P. Soler, Fencing in the Swampland: Quantum gravity constraints on large field inflation, J. High Energy Phys. 10 (2015) 023, arXiv:1503.04783 H. Matsui and F. Takahashi, Eternal inflation and Swampland conjectures, Phys.Rev. D 99, 023533 (2019), arXiv:1807.11938, I. Ben-Dayan, Draining the Swampland, arXiv:1808.01615 W. H. Kinney, S. Vagnozzi, and L. Visinelli, The zoo plot meets the Swampland: Mutual (in)consistency of single-field inflation, string conjectures, and cosmological data, arXiv:1808.06424

[13] M. Dias, J. Frazer, A. Retolaza, and A. Westphal, Primordial gravitational waves and the Swampland, Fortschr. Phys. 67, 1800063 (2019), arXiv:1807.06579.

[14] P. Agrawal, J. Fan, and M. Reece, Clockwork axions in cosmology: is chromonatural inflation chrononatural?, J. High Energy Phys. 10 (2018) 193, arXiv:1806.09621.

[15] A. Achcarro and G. A. Palma, The string Swampland constraints require multi-field inflation, J. Cosmol. Astropart. phys. 02 (2019) 041, arXiv:1807.04390

[16] A. Kehagias and A. Riotto, A note on inflation and the Swampland, Fortschr. Phys. 66, 1800052 (2018), arXiv:1807.05445

[17] Y. Akrami, R. Kallosh, A. Linde, and V. Vardanyan, The landscape, the Swampland and the era of precision cosmology, Fortschr. Phys. 67, 1800075 (2019), arXiv: 1808.09440

[18] J. P. Conlon, The de Sitter Swampland conjecture and supersymmetric AdS vacua, Int. J. Mod. Phys. A 33, 1850178 (2018), arXiv:1808.05040.

[19] A. Berera, Warm Inflation, Phys. Rev. Lett. 75, 3218 (1995), arXiv:astro-ph/9509049

[20] A. Berera, I. G. Moss, and R. O. Ramos, Warm inflation and its microphysical basis, Rep. Prog. Phys. 72, 026901 (2009), arXiv:0808.1855

[21] M. Bastero-Gil and A. Berera, Warm inflation model building, Int. J. Mod. Phys. A 24, 2207 (2009), arXiv:0902.0521

[22] S. Das, A note on single-field inflation and the Swampland criteria, arXiv:1809.03962.

[23] R. Allahverdi, R. Brandenberger, F. Y. Cyr-Racine, and A. Mazumdar, Reheating in inflationary cosmology: Theory and applications, Annu. Rev. Nucl. Part. Sci. 60, 27 (2010), arXiv:1001.2600

[24] M. A. Amin, M. P. Hertzberg, D. I. Kaiser, and J. Karouby, Nonperturbative dynamics of reheating after inflation: A review, Int. J. Mod. Phys. D 24, 1530003 
(2014), arXiv:1410.3808

[25] M. Bastero-Gil, A. Berera, and R. O. Ramos, Dissipation coefficients from scalar and fermion quantum field interactions, J. Cosmol. Astropart. Phys. 09 (2011) 033, arXiv:1008.1929.

[26] M. Bastero-Gil, A. Berera, R. O. Ramos, and J. G. Rosa, General dissipation coefficient in low-temperature warm inflation, J. Cosmol. Astropart. Phys. 01 (2013) 016, arXiv:1207.0445

[27] L. M. H. Hall, I. G. Moss, and A. Berera, Scalar perturbation spectra from warm inflation, Phys. Rev. D 69, 083525 (2004), arXiv:astro-ph/0305015

[28] C. Graham and I. G. Moss, Density fluctuations from warm inflation, J. Cosmol. Astropart. Phys. 07 (2009) 013, arXiv:0905.3500.

[29] M. Bastero-Gil, A. Berera, and R. O. Ramos, Shear viscous effects on the primordial power spectrum from warm inflation, J. Cosmol. Astropart. Phys. 07 (2011) 030, arXiv:1106.0701.

[30] M. Bastero-Gil, A. Berera, I. G. Moss, and R. O. Ramos, Cosmological fluctuations of a random field and radiation fluid, J. Cosmol. Astropart. Phys. 05 (2014) 004, arXiv:1401.1149.

[31] M. Bastero-Gil, A. Berera, I. G. Moss, and R. O. Ramos, Theory of non-Gaussianity in warm inflation, J. Cosmol. Astropart. Phys. 12 (2014) 008, arXiv:1408.4391

[32] L. Visinelli, Cosmological perturbations for an inflaton field coupled to radiation, J. Cosmol. Astropart. Phys. 01 (2015) 005, arXiv:1410.1187.

[33] M. Benetti and R. O. Ramos, Warm inflation dissipative effects: Predictions and constraints from the Planck data, Phys. Rev. D 95, 023517 (2017), arXiv:1610.08758.

[34] R. O. Ramos and L. A. da Silva, Power spectrum for inflation models with quantum and thermal noises, J. Cosmol. Astropart. Phys. 03 (2013) 032, arXiv:1302.3544.

[35] X. B. Li, H. Wang, and J. Y. Zhu, Gravitational waves from warm inflation, Phys. Rev. D 97, 063516 (2018), arXiv:1803.10074.

[36] M. Bastero-Gil, A. Berera, R. O. Ramos, and J. G. Rosa, Warm Little Inflaton, Phys. Rev. Lett. 117, 151301 (2016), arXiv:1604.08838.

[37] A. Berera, M. Gleiser, and R. O. Ramos, Strong dissipative behavior in quantum field theory, Phys. Rev. D 58, 123508 (1998), arXiv:hep-ph/9803394

[38] A. Berera, M. Gleiser, and R. O. Ramos, A First principles warm inflation model that solves the cosmological horizon / flatness problems, Phys. Rev. Lett. 83, 264 (1999), arXiv:hep-ph/9809583

[39] Y. Zhang, Warm inflation with a general form of the dissipative coefficient, J. Cosmol. Astropart. Phys. 03 (2009) 023, arXiv:0903.0685, R. Herrera, M. Olivares, and N. Videla, General dissipative coefficient in warm intermediate inflation in loop quantum cosmology in light of Planck and BICEP2, Int. J. Mod. Phys. D 23, 1450080 (2014), arXiv:1404.2803 A. Jawad, S. Hussain, S. Rani, and N. Videla, Impact of generalized dissipative coefficient on warm inflationary dynamics in the light of latest Planck data, Eur. Phys. J. C 77, 700 (2017), arXiv:1709.10430.

[40] Y. L. Bolotin, A. Kostenko, O. A. Lemets, and D. A. Yerokhin, Cosmological evolution with interaction between dark energy and dark matter, Int. J. Mod. Phys. D 24, 1530007 (2014), arXiv:1310.0085.

[41] C. M. S. Barbosa, H. Velten, J. C. Fabris, and
R. O. Ramos, Assessing the impact of bulk and shear viscosities on large scale structure formation, Phys. Rev. D 96, 023527 (2017), arXiv:1702.07040

[42] Y. Akrami et al., (Planck Collaboration), Planck 2018 results. X. Constraints on inflation, arXiv:1807.06211.

[43] M. Bastero-Gil, A. Berera, R. O. Ramos, and J. G. Rosa (work in progress).

[44] D. H. Lyth, What would we learn by detecting a gravitational wave signal in the cosmic microwave background anisotropy?, Phys. Rev. Lett. 78, 1861 (1997), arXiv:hep$\mathrm{ph} / 9606387$.

[45] S. Brahma and M. Wali Hossain, Avoiding the string Swampland in single-field inflation: Excited initial states, arXiv:1809.01277

[46] C. Germani, L. Martucci, and P. Moyassari, Introducing the Slotheon: A slow Galileon scalar field in curved spacetime, Phys. Rev. D 85103501 (2012), arXiv:1108.1406.

[47] M. C. Bento, R. G. Felipe, and N. M. C. Santos, Brane assisted quintessential inflation with transient acceleration, Phys. Rev. D 77, 123512 (2008), arXiv:0801.3450.

[48] M. A. Cid, S. del Campo, and R. Herrera, Warm inflation on the brane, J. Cosmol. Astropart. Phys. 10 (2007) 005, arXiv:0710.3148

[49] C. M. Lin, K. W. Ng, and K. Cheung, Chaotic inflation on the brane and the Swampland criteria, arXiv:1810.01644

[50] T. Kobayashi, M. Yamaguchi, and J. Yokoyama, Generalized $G$ inflation: Inflation with the most general secondorder field equations, Prog. Theor. Phys. 126, 511 (2011), arXiv:1105.5723

[51] R. Herrera, S. del Campo, and C. Campuzano, Tachyon warm inflationary universe models, J. Cosmol. Astropart. Phys. 10 (2006) 009, arXiv:astro-ph/0610339 Y. F. Cai, J. B. Dent, and D. A. Easson, Warm DBI inflation, Phys. Rev. D 83, 101301 (2011), arXiv:1011.4074 X. M. Zhang and j. Y. Zhu, Extension of warm inflation to noncanonical scalar fields, Phys. Rev. D 90, 123519 (2014), arXiv:1402.0205. M. Motaharfar, E. Massaeli, and H. R. Sepangi, Power spectra in warm $G$ inflation and its consistency: Stochastic approach, Phys. Rev. D 96, 103541 (2017), arXiv:1705.04049 R. Herrera, GWarm inflation, J. Cosmol. Astropart. Phys. 05 (2017) 029, arXiv:1701.07934 M. Motaharfar, E. Massaeli, and H. R. Sepangi, Warm Higgs $G$ inflation: Predictions and constraints from Planck 2015 likelihood, J. Cosmol. Astropart. Phys. 10 (2018) 002, arXiv:1807.09548

[52] A. Berera, Warm inflation at arbitrary adiabaticity: A Model, an existence proof for inflationary dynamics in quantum field theory, Nucl. Phys. B585, 666 (2000), arXiv:hep-ph/9904409

[53] S. Bartrum, M. Bastero-Gil, A. Berera, R. Cerezo, R. O. Ramos, and J. G. Rosa, The importance of being warm (during inflation), Phys. Lett. B 732, 116 (2014), arXiv:1307.5868 A. Berera, J. Mabillard, M. Pieroni, and R. O. Ramos, Identifying universality in warm inflation, J. Cosmol. Astropart. Phys. 07 (2018) 021, arXiv:1803.04982 L. L. Graef and R. O. Ramos, Probability of warm inflation in loop quantum cosmology, Phys. Rev. D 98, 023531 (2018), arXiv:1805.05985, M. BasteroGil, A. Berera, R. Hernandez-Jimenez, and J. G. Rosa, Dynamical and observational constraints on the Warm Little Inflaton scenario, arXiv:1805.07186.

[54] M. Bastero-Gil, S. Bhattacharya, K. Dutta, and M. R. Gangopadhyay, Constraining warm inflation with 
CMB data, J. Cosmol. Astropart. Phys. 02 (2018) 054, arXiv:1710.10008; R. Arya, A. Dasgupta, G. Goswami, J. Prasad, and R. Rangarajan, Revisiting CMB constraints on warm inflation, J. Cosmol. Astropart. Phys. 02 (2018) 043, arXiv:1710.11109.

[55] H. Ooguri, E. Palti, G. Shiu, and C. Vafa, Distance and de Sitter conjectures on the Swampland, Phys. Lett. B
788, 180 (2019), arXiv:1810.05506

[56] S. Das, Warm inflation in the light of Swampland criteria, arXiv:1810.05038

[57] M. Bastero-Gil, A. Berera, R. Hernández-Jiménez, and J. G. Rosa, Warm inflation within a supersymmetric distributed mass model, arXiv:1812.07296. 